\title{
PREDICCIÓN DE LA IDEACIÓN SUICIDA DESDE LA PERCEPCIÓN DE LA CRIANZA PARENTAL EN ADOLESCENTES YUCATECOS
}

PREDICTION OF SUICIDAL IDEATION FROM THE PERCEPTION

OF PARENTING IN YUCATECAN ADOLESCENTS

Lourdes Cortés Ayala*, Julio Isaac Vega Cauich*, José Alberto López Rodríguez**,

Sergio Daniel Garma Itzá**

Universidad Autónoma de Yucatán*, Universidad Marista de Mérida**, México

Correspondencia: julio.vega@correo.uady.mx

\section{RESUMEN}

El objetivo de este estudio fue identificar la incidencia de ideación suicida, la percepción de las prácticas de crianza parentales y los factores de la crianza de padres y madres que predicen la ideación suicida en adolescentes. Participaron 208 estudiantes de secundaria (52.4\% mujeres y 47.6\% hombres) seleccionados no probabilísticamente. Se aplicó la Escala de riesgo suicida y la Escala de percepción de prácticas parentales de crianza para adolescentes. Los resultados evidencian ideación suicida en $14 \%$ de la muestra estudiada; se obtiene que la calidez del padre, disciplina del padre y comunicación de la madre estuvieron asociadas de manera inversa con el riesgo de suicidio; un factor de riesgo fue el énfasis en el logro de la madre, ya que este factor estuvo asociado de manera significativa y directa con el riesgo de suicidio. Se discuten las implicaciones de los resultados para la prevención del suicidio en adolescentes. 
Palabras clave: ideación suicida, suicidio, percepción de prácticas de crianza, adolescentes, predicción.

\section{ABSTRACT}

The objective of this study was to identify the incidence of suicidal ideation, the perception of parenting practices and the factors of parenting that predict suicidal ideation in adolescents. 208 high school students $(52.4 \%$ women and $47.6 \%$ men) were selected in a nonrandom sample. The Suicidal Risk Scale and the Perception Scale of Parental Parenting Practices for Adolescents were applied. The results show suicidal ideation in $14 \%$ of the sample studied. Regarding the prediction models, the father's warmth, father's discipline and mother's communication were inversely associated with the risk of suicide; A risk factor was the emphasis on achievement by the mother, since this factor was significantly and directly associated with the risk of suicide. The implications of the results for suicide prevention in adolescents are discussed.

Keywords: suicidal ideation, suicide, perception of parenting practices, adolescents, prediction.

\section{INTRODUCCIÓN}

El suicidio constituye una expresión de violencia auto infringida, con un resultado fatal, anticipado (OMS, 2012) considerado un problema de salud pública a nivel mundial ya que anualmente el rango de suicidios va en aumento, siendo la segunda causa de muerte entre personas de 15 a 29 años (OMS, 2014). En México, de acuerdo con los últimos datos del Instituto Nacional de Estadística y Geografía, durante 2016 ocurrieron 6,291 suicidios, de los cuales ocho de cada 10 fueron cometidos por un hombre. En cuanto al volumen de suicidios, hay variación por zona geográfica y 
entidad federativa; Chihuahua y Yucatán duplican la tasa nacional con 11.4 y 10.2 suicidios por cada 100,000 habitantes respectivamente. Por lo que brindar información al respecto en lugares con alta prevalencia como Yucatán, resulta de relevancia para comprender mejor el fenómeno y obtener evidencia empírica para mejorar los programas de prevención.

El suicidio es un proceso complejo que inicia con la ideación suicida o preocupación autodestructiva, planificación de un acto letal y deseo de muerte, hasta concretarse en un acto consumado (Sánchez Sosa, VillarealGonzález, Musitu \& Martínez, 2010). De ahí que el estudio de la ideación suicida resulta relevante para propósitos de intervenciones primarias. La ideación suicida consiste en las ideas o intenciones de suicidio, esto es, los pensamientos de terminar con la propia vida con o sin el propósito de materializarlos (Robledo, 2007).

La ideación suicida ha mostrado ser un factor predictor del intento de suicidio y del suicidio consumado (Cabra, Infante \& Sosa, 2010; Córdoba, Cubillas \& Román 2011; Córdoba, Estrada \& Velázquez, 2013; Gibb, Beautrais \& Fergusson, 2005; Pérez, Ibañez \& Reyes, 2008; Toro, Avendaño \& Castrillón, 2016; Valdivia, Silva, Sanhueza, Cova \& Melipillán, 2015; Wichstrom, 2000; Nock, et al, 2008; Schilling, Aseltine, Glanovsky, James \& Jacobs, 2009; Urzúa \& Caqueo-Urízar, 2011). La evidencia señala que las niñas y adolescentes tienden más a las tentativas suicidas que los varones, aunque los varones adolescentes tienen cinco veces más probabilidades de daños físicos graves o muerte en sus intentos (Garlow, 2008; SánchezTeruel, 2012; Sánchez-Teruel, García-León \& Muela-Martínez, 2013; Sánchez, Cáceres \& Gómez, 2002; Rosales, Córdoba \& Fonseca, 2013). 
En este sentido, podríamos considerar a la ideación suicida como un factor de riesgo del intento de suicidio y el suicidio consumado considerando que la OMS define los factores de riesgo como cualquier rasgo, característica o exposición de un individuo que aumenta su probabilidad de sufrir una enfermedad o lesión. De forma específica, los factores de riesgo suicida se refieren a factores predisponentes que pueden desencadenar un acto suicida (Stengel, 1987), y según la OMS entre los más importantes están las emociones negativas y eventos estresantes (Pitman, Osborn, Rantell \& King, 2016; Rosales, Córdoba \& Guerrero, 2013) así como problemas familiares (Quintanilla-Montoya et al, 2015).

Comprender los factores de riesgo y protección asociados al comportamiento suicida resulta esencial para su atención. Así, algunos estudios han encontrado que los factores familiares se encuentran entre los tres principales factores asociados a la ideación suicida junto con variables psicológicas y socioculturales (Kim \& Lee, 2009). Varias investigaciones evidencian el papel de la disfuncionalidad familiar como factor de riesgo de la ideación y conducta suicida (Aguirre-Flores et al, 2014; Bahamon \& Alarcon, 2018; Toro, Paniagua, González \& Montoya, 2006; Loboa \& Morales, 2016), sobre todo cuando se caracteriza por el desapego en el escenario familiar, sentirse enojado, sentirse triste, solo, deprimido e incomprendido por la familia (Chávez-Hernández, González-Forteza, Juárez, Vázquez \& Jiménez, 2015).

La investigación también ha comprobado desde tiempo atrás que la ideación es un indicador de riesgo suicida (McAuliffe, 2002; ChávezHernández, et al, 2015). De acuerdo con Sánchez-Teruel y Robles Bello (2014) aunque existe numerosa literatura para identificar factores de riesgo para la ideación y la conducta suicida, es escasa la enfocada al estudio 
de los factores protectores, en adolescentes. Además, los pocos intentos sistematizados como el de Kim y Lee (2009) se enfocan en revisiones sistemáticas de países asiáticos. Sin embargo, las aproximaciones empíricas existentes han encontrado algunos factores de protección importantes a nivel familiar. En esta línea, Florenzano, et al (2011) encuentran como factores protectores a la aceptación parental, la promoción de la autonomía psicológica parental, la calidad de la relación con la madre y con el padre, la amabilidad parental, expresión de afectos físicos, monitoreo paterno y participación en decisiones familiares. Otros estudios señalan el papel protector de las relaciones estrechas con la familia (Kim \& Lee, 2009) y la percepción de apoyo familiar (Mustanski \& Liu, 2013; Rhodes et al, 2012). La percepción de funcionalidad familiar también constituye un factor protector (Forero, Sibato \& Salamanca, 2018; Morales et al 2017), así como la cohesión familiar (Cabra, Infante \& Sossa, 2010).

Dado que el suicido consumado, como proceso, se inicia con la ideación, la investigación en este tema resulta de particular importancia para su prevención. A partir de la alta prevalencia de suicidios en el estado de Yucatán y la escasa información sobre el papel protector de la familia a nivel local, el conocimiento actual obliga a realizar más investigación en este contexto sociocultural para su eventual prevención. Por ello, el objetivo de este estudio es determinar la prevalencia de ideación suicida, la percepción de prácticas parentales de crianza, así como las diferencias en esta variable en función del riesgo de ideación suicida, e identificar los factores de la percepción de crianza paterna y materna que predicen la ideación suicida, en adolescentes. 


\section{MÉTODO}

\section{Participantes}

Participaron 208 adolescentes, 52.4\% mujeres ( $\mathrm{N}=109)$ y $47.6 \%$ hombres $(\mathrm{N}=99)$, seleccionados no probabilísticamente, estudiantes de secundaria de dos escuelas privadas de la ciudad de Mérida, México, con un rango de edad de 12 a 15 años ( $M=13.19$ - D. E.=.597) El 28\% (59) estudiaba el primer grado y $71.6 \%$ (149) el segundo grado de secundaria. El 90 \% vivía con ambos padres, $9 \%$ solo con su papá y $1 \%$ solo con su mamá.

\section{Instrumentos}

Escala de riesgo suicida (Plutchik, Van Praag, Conte \& Picard, 1989) en su versión española (Rubio, et al 1998). La escala está conformada por 15 ítems con respuesta dicotómica ( $\mathrm{si} / \mathrm{no}$ ). Cada respuesta afirmativa puntúa uno y cada respuesta negativa es igual a cero puntos. El rango total posible oscila 0 a 15 puntos. Esta versión reporta una confiabilidad test-restest de 0.89; y una consistencia interna de 0.89 también. Para este estudio se reporta una consistencia interna de .76 según el estadístico Omega de McDonald. Los autores de la versión española proponen un punto de corte de 6 puntos, donde una puntuación superior a seis puntos indica riesgo de suicidio y presencia de ideación suicida. Cuanto mayor es la puntuación mayor es el riesgo. La escala y este punto de corte ha mostrado evidencia de validez de criterio al poder clasificar correctamente entre pacientes control y con intentos de suicidio previo mostrando una sensibilidad de 0.74 y una especificidad de 0.95. Por su parte, estudios de validación en muestras mexicanas con respecto a la validez de constructo señalan que la escala puede tener al menos cuatro factores que explican el $52.64 \%$ de la varianza: 1) sentimientos de inutilidad; 2) ideación suicida; 3 ) desesperanza, y 4) factores sociales (Santana-Campas \& Santoyo Telles, 2018). 
Escala de Percepción de Prácticas Parentales de Crianza para Adolescentes (EPPPCA) (Flores, Cortés, Morales y Campos, 2017). Consta de 26 reactivos en un formato tipo Likert de cinco opciones de respuesta que mide la frecuencia en la que los adolescentes perciben que sus padres realizan diversas conductas para educarlos. El instrumento está dividido en dos sub escalas, una para madres y otra para padres, y ambas mide las siguientes dimensiones: (a) Comunicación, (seis ítems) se refiere al intercambio de información verbal entre padres e hijos que permite conectar los sentimientos de ambos, y hacer sentir a estos últimos cuidados, respetados, importantes y apoyados $(=.91)$; (b) Calidez (tres ítems), es la expresión verbal y conductas explicitas de aceptación, apoyo, afecto e interés hacia las necesidades, emociones y preocupaciones de los hijos que crean un ambiente en el cual la relación con los hijos fluyen en forma agradable y positiva $(=.88$ ); (c) Énfasis en el logro (cinco ítems) indica el nivel de exigencia y presión de los padres hacia los hijos para el logro de altos niveles de madurez, autonomía y competencia en diversas actividades, basado en la confianza en las capacidades de los hijos, fomentando el pensamiento crítico, la perseverancia y el destacar ( $=.86$ ); (d) Supervisión y monitoreo (cuatro ítems) se refiere al conductas parentales que involucran atención y supervisión de las actividades de los hijos, indagando acerca de lo que hacen, en dónde se encuentran y/o con quienes andan, por medio de los propios hijos o por medio de otras fuentes ( = .79); (e) Apoyo escolar (cuatro ítems), es el acompañamiento, guía y apoyo emocional e instrumental que los padres ofrecen a los hijos para sus actividades escolares, que incluye la motivación para que realicen adecuadamente sus responsabilidades escolares y logren éxito académico ( = .81), y (f) Disciplina, límites y reglas (cuatro ítems) son las estrategias -positivas y negativas- que utilizan los padres para hacer que sus hijos 
se comporten y cumplan con sus expectativas y con las reglas que han impuesto en la casa $(=.73)$.

Los estudios previos señalan evidencia de validez de constructo ya que ha permitido identificar prácticas de crianza materna diferenciadas entre hijos e hijas. Por ejemplo, los resultados obtenidos con esta escala son consistentes con elementos socioculturales de la cultura mexicana, ya que las mujeres suelen reportar mayores niveles de supervisión y monitoreo por parte de la madre en comparación con los hombres (Flores, Cortés, Morales, Campos \& Díaz-Loving, 2019).

\section{Procedimiento}

Primeramente, se obtuvieron los permisos correspondientes en cada uno de los centros educativos, previa explicación de los objetivos del estudio, para la recolección de la información. La administración de las escalas se realizó de manera colectiva en los salones de clase de las diferentes instituciones seleccionadas y en presencia de los profesores respectivos. De acuerdo con el Código Ético del Psicólogo (2002), a los participantes se les invitó a colaborar en el estudio, explicando su objetivo. Se les indicó que la participación era voluntaria, y por tanto que podrían retirarse cuando desearan sin alguna consecuencia. Asimismo, se hizo énfasis en el carácter anónimo y confidencial de las respuestas, por lo que podrían contestar sin temor a ser identificados ya que los datos se procesarían de forma grupal por medio de un software. Los participantes firmaron cartas de consentimiento informado antes de responder los instrumentos.

Los datos finalmente fueron analizados con el paquete estadístico libre JAMOVI (2020) en su versión 1.2. Primeramente, se realizaron estadísticos descriptivos de las dos escalas utilizadas, así como de su consistencia 
interna. Posteriormente, se realizaron descriptivos sobre la Percepción de Prácticas Parentales de Crianza tanto en el grupo de riesgo como sin riesgo de suicidio, así como las respectivas comparaciones de cada dimensión, utilizando la prueba $\mathrm{U}$ de Mann-Whitney ya que las puntuaciones no tuvieron una distribución normal. Finalmente, para analizar las variables que estaban asociadas al riesgo de suicidio se realizó una regresión logística binaria utilizando como variable dependiente la categoría de riesgo de suicidio y como variables predictoras cada una de las dimensiones de las escalas de crianza parental, y como variable de control el sexo de los participantes para eliminar el posible efecto del sexo tanto en la variable predicha como en las predictoras. Todos los análisis inferenciales fueron realizados considerando una alfa de .05 .

\section{RESULTADOS}

Primeramente, los resultados obtenidos a partir de los puntos de corte de seis puntos o más, señala que un $15 \%(\mathrm{n}=31)$ de la muestra presenta un riesgo de suicidio; y mientras que un $85 \%(\mathrm{n}=177)$ no presenta riesgo de suicidio. Con respecto a la Percepción de Prácticas Parentales de Crianza, los resultados generales se observan en la Tabla 1, donde se muestra que en general los factores de la madre suelen puntuar superiores a los del padre, aunque en ambos casos los puntajes son relativamente altos. Además, los niveles de consistencia interna pueden ser considerados de aceptables a buenos, ya que los valores tanto del alfa de Cronbach como el Omega de McDonald en su mayoría fueron superiores a .70, aunque algunas dimensiones tuvieron valores relativamente bajos, estos pueden ser considerados adecuados en consideración de que matemáticamente las medidas de consistencia interna subestiman la confiabilidad verdadera (Lord \& Novick, 1968). 
Tabla 1.

Descriptivos y consistencia interna para los factores de Prácticas Parentales de Crianza de padre y madre.

\begin{tabular}{|c|c|c|c|c|}
\hline \multirow[b]{2}{*}{ Dimensiones } & \multirow[b]{2}{*}{ Media } & \multirow[b]{2}{*}{ D.E. } & \multicolumn{2}{|c|}{ de Omega } \\
\hline & & & Cronbach & McDonald \\
\hline \multicolumn{5}{|l|}{ Factores del padre } \\
\hline Comunicación & 3.82 & 1.09 & .899 & .900 \\
\hline Calidez & 4.06 & 1.07 & .866 & .868 \\
\hline Énfasis en el logro & 4.22 & .865 & .820 & .828 \\
\hline Supervisión y monitoreo & 4.07 & .915 & .735 & .755 \\
\hline Apoyo escolar & 3.86 & 1.01 & .756 & .767 \\
\hline Disciplina, límites y reglas & 3.75 & 1.01 & .753 & .755 \\
\hline \multicolumn{5}{|l|}{ Factores de la madre } \\
\hline Comunicación & 4.21 & .856 & .877 & .881 \\
\hline Calidez & 4.48 & .792 & .845 & .848 \\
\hline Énfasis en el logro & 4.42 & .678 & .757 & .786 \\
\hline Supervisión y monitoreo & 4.52 & .559 & .493 & .532 \\
\hline Apoyo escolar & 4.30 & .723 & .689 & .698 \\
\hline Disciplina, límites y reglas & 4.04 & .863 & .728 & .732 \\
\hline
\end{tabular}

\section{Comparaciones entre grupos}

Posteriormente, también se contrastaron las dimensiones de Percepción de Prácticas Parentales de Crianza entre los grupos con y sin riesgo de suicidio. Tal como se observa en la Tabla 2, el grupo sin riesgo de suicidio presenta puntuaciones más altas en todas las dimensiones, con diferencias estadísticamente significativas en las dimensiones de Comunicación del padre, Calidez del padre, Apoyo escolar del padre, Comunicación de la madre, Calidez de la madre, y Apoyo escolar de la madre. Se calculó el tamaño del efecto a través de la $d$ de Cohen (1989) para conocer la magnitud de este al analizar el efecto de la variable riesgo de suicidio (ideación suicida). De acuerdo con la teoría de Cohen puntuaciones inferiores a .20 indican un tamaño del efecto despreciable, entre .21 a .49 el tamaño del efecto es pequeño, de .50 a .79 es mediano y de .80 en adelante es grande. Como puede observarse en la Tabla 2, para los padres, en las Comunicación y Calidez se obtiene un tamaño del efecto mediano, 
y para las dimensiones de Apoyo escolar, y Disciplina, límites y reglas, el tamaño del efecto es pequeño. En el caso de la sub escala de mamá, para las tres dimensiones, Calidez, Apoyo escolar y Comunicación, el tamaño del efecto es mediano.

Tabla 2 .

Diferencias para los factores de padres y madres por grupo con y sin riesgo de ideación suicida.

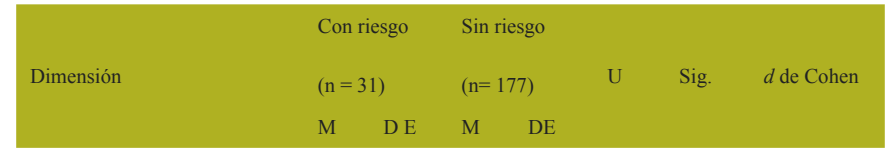

Factores del padre

\begin{tabular}{|lrrrrrrrr}
\hline Comunicación & 3.25 & 1.285 & 3.92 & 1.032 & 1883 & .005 & .630 \\
\hline Calidez & 3.41 & 1.270 & 4.18 & .994 & 1712 & $<.001$ & .734 \\
\hline Énfasis en el logro & 3.94 & 1.043 & 4.27 & .824 & 2242 & .101 & .379 \\
\hline Supervisión y monitoreo & 3.91 & 1.098 & 4.10 & .880 & 2585 & .605 & .201 \\
\hline Apoyo escolar & 3.50 & 1.147 & 3.93 & .977 & 2127 & .045 & .429 \\
\hline Disciplina, límites y reglas & 3.40 & 1.150 & 3.81 & .975 & 2140 & .050 & .411 \\
\hline
\end{tabular}

Factores de la madre

\begin{tabular}{|lcccccccc|}
\hline Comunicación & 3.68 & .978 & 4.30 & .801 & 1613 & $<.001$ & .747 \\
\hline Calidez & 4.12 & .957 & 4.54 & .746 & 1940 & .005 & .531 \\
\hline Énfasis en el logro & 4.30 & .825 & 4.44 & .649 & 2499 & .421 & .194 \\
\hline Supervisión y monitoreo & 4.46 & .604 & 4.53 & .552 & 2633 & .712 & .113 \\
\hline Apoyo escolar & 3.98 & .856 & 4.36 & .684 & 2011 & .016 & .532 \\
\hline Disciplina, límites y reglas & 3.97 & 1.127 & 4.05 & .812 & 2603 & .647 & .094 \\
\hline
\end{tabular}

\section{Modelos estadísticos}

Finalmente, para analizar las variables que estaban asociadas al riesgo de suicidio se realizó una regresión logística. El modelo resultante no tuvo problemas de multicolinealidad y tuvo un porcentaje de explicación de varianza de $29.3 \%$, con un área bajo la curva de $81.8 \%$ lo que señala una clasificación adecuada. Los resultados sobre las variables predictoras se observan en la Tabla 3, donde se aprecia que la calidez del padre, disciplina del padre y comunicación de la madre estuvieron asociadas de 
manera inversa con el riesgo de suicidio, es decir que a mayor nivel de estos factores menos riesgo de suicidio. De estas variables, la que presentó un mayor efecto según la odds ratio fue la Comunicación de la madre, seguida de la calidez del padre y por último la disciplina del padre. En contraste una variable que fue un factor de riesgo fue el énfasis en el logro, de la madre, ya que esta estuvo asociada de manera significativa y directa con el riesgo de suicidio.

Tabla 3 .

Predicción de la ideación suicida en función de la Percepción de Prácticas Parentales de Crianza.

\begin{tabular}{|c|c|c|c|c|c|}
\hline \multirow[b]{2}{*}{ Predictor } & \multirow{3}{*}{$\begin{array}{l}\text { Parámetro } \\
-.124\end{array}$} & \multirow{3}{*}{$\begin{array}{l}\text { Sig. } \\
.950\end{array}$} & \multirow{3}{*}{$\begin{array}{l}\text { Odds ratio } \\
.883\end{array}$} & \multicolumn{2}{|c|}{ Intervalo de Confianza (95\%) } \\
\hline & & & & Inferior & Superior \\
\hline Intercepto & & & & .018 & 42.604 \\
\hline \multicolumn{6}{|l|}{ Padre } \\
\hline Comunicación & .473 & .249 & 1.605 & .718 & 3.586 \\
\hline Calidez & -1.165 & .005 & .312 & .137 & .707 \\
\hline Énfasis en el logro & .299 & .551 & 1.349 & .505 & 3.601 \\
\hline Supervisión y monitoreo & .510 & .187 & 1.666 & .781 & 3.553 \\
\hline Apoyo escolar & .454 & .266 & 1.575 & .706 & 3.510 \\
\hline Disciplina & -.874 & .033 & .417 & .187 & .930 \\
\hline \multicolumn{6}{|l|}{ Madre } \\
\hline Comunicación & -1.777 & $<.001$ & .169 & .062 & .455 \\
\hline Calidez & .628 & .132 & 1.874 & .827 & 4.244 \\
\hline Énfasis en el logro & 1.389 & .018 & 4.013 & 1.272 & 12.655 \\
\hline Supervisión y monitoreo & -.276 & .628 & .759 & .248 & 2.319 \\
\hline Apoyo escolar & -.917 & .055 & .400 & .156 & 1.021 \\
\hline Disciplina & .755 & .072 & 2.127 & .934 & 4.839 \\
\hline \multicolumn{6}{|l|}{ Sexo } \\
\hline Hombre - Mujer & -.711 & .146 & .491 & .188 & 1.280 \\
\hline
\end{tabular}




\section{DISCUSIÓN}

Este estudio tuvo como objetivos determinar la prevalencia de la ideación suicida, la percepción de las prácticas de crianza paternas en adolescentes, en función del riesgo suicida y por último identificar los factores de percepción de la crianza que permiten predecir la ideación suicida en adolescentes. Respecto a la prevalencia, los resultados indicaron que $14.9 \%$ de participantes presenta ideación suicida, cifra que es ligeramente superior a la reportada por el estudio de Pinto et al (2009) con adolescentes yucatecos (13.15\%), y muy superior a las cifras reportadas para México por Rosales, Córdoba y Guerrero (2013), quienes encuentran que 5.5\% de chicos y $5.4 \%$ de chicas mexicanos presentaban ideación suicida. De igual forma, es superior a estudios más recientes con representatividad nacional como la realizada por la ENCODAT (Encuesta Nacional de Consumo de Drogas, Alcohol y Tabaco) donde un 2.3\% presentó ideación suicida a nivel nacional y un $2.5 \%$ en Yucatán (Borges, Orozco, Villatoro, Medina-Mora, Fleiz \& Díaz-Salazar, 2019). Los estudios anteriores reflejan el aumento en las tasas de suicidio en Yucatán durante los últimos años (Balam, 2019).

Respecto a la percepción de la crianza parental, los datos generales muestran que los adolescentes tienen una percepción positiva de las prácticas de crianza de ambos padres, caracterizada por expresiones de afecto y calidez, cuidados a través de la preocupación y monitoreo de las actividades de los hijos y expresiones de motivación al logro escolar brindándoles el apoyo necesario. Sin embargo, los hijos e hijas valoran más a la madre en todas las dimensiones especialmente en la supervisión y monitoreo y en la expresión de afectos. Estos resultados coinciden con estudios previos con adolescentes yucatecos, (Flores \& Cortés, 2017; Cortés, Flores \& Morales, 2017; Casáis \& Flores, 2017) y con otros estudios (Lai \& McBride-Chang, 2001; Morales et al, 2014; Parker \& 
McNally, 2008) que han mostrado el papel central de la madre en la crianza y socialización, ya que parece ser que sigue siendo la encargada principal, están más implicadas en la crianza por lo que tienen un lugar más relevante en la educación de sus hijos (Capano, Gonzáles \& Massonier, 2016; Etxebarria, Apodaca, Fuentes, López \& Ortiz, 2009; Parra \& Oliva, 2002; Tur-Porcar, Mestre, Samper \& Malonda, 2012). Por ello en este estudio, en primer término, es valorada con un alto monitoreo y muy afectuosa; el perfil obtenido para el padre es muy similar al de la madre, aunque más moderado. Estos resultados coinciden con investigaciones que señalan a la aceptación parental, la promoción de la autonomía psicológica parental, la calidad de la relación con la madre y con el padre, la amabilidad parental, expresión de afectos físicos y monitoreo, como recursos protectores con los que cuentan estos jóvenes ante diversas condiciones adversas como la ideación suicida (Florenzano, et al 2011; Mustanski \& Liu, 2013; Rhodes et al, 2012). En esta línea, estilos de crianza afectuosos promueven en los hijos el desarrollo de conductas prosociales y autorregulación emocional que constituyen factores protectores contra la depresión (Florenzano, et al 2010).

Cuando el análisis de la crianza se hace en función de la ideación vs. no ideación suicida, los resultados indican que quienes si tienen ideación suicida perciben menor calidez y comunicación por parte del padre y madre, así como menos apoyo escolar e imposición de disciplina, límites y reglas por parte del padre. Asimismo, reciben importante apoyo de ambos en las tareas y actividades escolares y supervisando sus calificaciones y desempeño académico, aunque el tamaño del efecto obtenido para el padre es pequeño y para la madre es mediano. Sin embargo, si atendemos a las diferencias percibidas entre ambos padres, los chicos sin ideación valoran más el afecto, la comunicación y el apoyo escolar recibido de la madre, 
donde se obtiene que el tamaño del efecto en todas las dimensiones es mediano. Los adolescentes del grupo sin ideación perciben que el padre les disciplina al explicarles las reglas de la casa, y castigarles si no las cumplen o no obedecen e indicarles cómo deben comportarse, aunque para esta dimensión el tamaño del efecto es pequeño. Los resultados coinciden con la literatura donde se enfatiza el importante papel de la familia, el apoyo percibido de padres y madres, así como expresión de afectos físicos, y monitoreo paterno como factores protectores ante la ideación suicida (Cabra, et al, 2010; Forero, et al, 2018; Morales et al 2017; Mustanski \& Liu, 2013; Rhodes et al, 2012).

Por último, las variables que predicen la ideación suicida son la comunicación con la madre y la calidez y disciplina del padre, de ahí que la percepción de una madre con quien se puede hablar de cualquier tema, interesada, atenta y que les reconforta cuando están triste contribuye a un menor riesgo de ideación suicida y por tanto es un recurso protector importante, conjuntamente con la percepción de un padre afectuoso y cálido pero que les impone disciplina, límites y reglas para el propio bienestar de los adolescentes. Estas dimensiones conforman parte de estilos de crianza positivos que han demostrado ofrecer experiencias y escenarios familiares que promueven en los adolescentes conductas saludables para poner en práctica en diversos contextos y situaciones (Jacob Foundation, Hanbury \& Malti, 2011). Morales et al (2014) encuentran que la percepción de un padre sobreprotector aun con bajos niveles de cuidado se asocia a menor riesgo de ideación. En tanto que entornos familiares percibidos como desprotectores, con escaso control y apoyo positivo hacen aumentar los factores de riesgo al impedir el desarrollo de conductas saludables (Andrade, Bonilla \& Valencia, 2010; Fuentes, García, Gracia y Alarcón, 2015) y elevan los riesgos de suicidio (Pérez, et al 2013, Vallejo, Osorno 
\& Mazadiego, 2008). Andrade y Gonzáles (2017) y Morales, et al (2014) encuentran que cuando los padres son indulgentes o negligentes el riesgo de suicido se incrementa.

Por último, los resultados señalan que el énfasis en el logro de la madre estuvo asociado de manera significativa y directa con el riesgo de suicidio. Esta dimensión se refiere al nivel de exigencia y presión hacia los hijos para que logren ciertos estándares de madurez, autonomía y competencia personal que la madre considera deseables; si la percepción del hijo es que estas demandas son muy elevadas pueden generar malestar, incomodidad, frustración, tristeza e incluso enojo hacia sí mismo por no poder complacer a la madre o por no sentirse con la competencia para lograr lo que se le exige. Esto es consistente con los estudios que señalan que varios aspectos relacionados al perfeccionismo, incluidos las críticas y expectativas parentales, están asociados tanto a la ideación suicida como al intento de suicidio (Smith et al., 2018). Algunos estudios señalan que esta relación es mediada por la mala regulación emocional que pueden tener algunos individuos (Zeifman, Antony \& Kuo, 2020), pues el perfeccionismo ha sido asociado con trastornos afectivos como el trastorno depresivo mayor (Say, 2020). Por su parte, el hecho de que el énfasis en el logro por parte de la madre y no por parte del padre haya resultado significativo, coincide con la literatura que muestra que culturalmente se percibe a las madres como más sobre-controladoras e intrusivas que a los padres, ya que generalmente están a cargo del cuidado y de regular los eventos y las conductas cotidianas de sus hijos, por ello las madres pueden interferir más en el desarrollo de ciertas competencias de los hijos, que constituyen factores protectores, como la autonomía y la autoregulación (Lai \& McBride-Chang, 2001; Morales, et al 2014; Parker \& McNally, 2008). Los resultados también se explican a partir de los estudios que muestran que convivir con una madre/ 
padre autoritario, con elevados niveles de coerción física y verbal y baja implicación positiva aumenta el riesgo de suicidio (Andrade y Gonzáles, 2017; Andrade, 2012; Sánchez-Teruel \& Robles-Bello, 2014). Estudios realizados con adolescentes encuentran que la frecuencia de conflictos con la madre juega un papel relevante en la ideación suicida, (Aguilar, Sarmiento, Valencia \& Romero, 2007; González, Ramos, Caballero \& Wagner, 2003; Sarmiento \& Aguilar Villalobos, 2011). También han mostrado que sentirse enojado, triste, solo, deprimido o incomprendido en la familia puede generar sentimientos de culpa e inferioridad asociados a ideación suicida (Quintanilla-Montoya et al 2015; Hernández, de los Reyes, Gonzáles y Ptrats, 2011).

En conclusión, la ideación suicida como predictora del suicidio constituye un serio problema social y de salud pública, sin embargo, su naturaleza multicausal requiere analizar esta problemática a partir de factores personales, familiares y sociales. Los resultados obtenidos evidencian el rol predictivo de la crianza positiva basada en la comunicación, afecto y demandas de madurez moderadas y racionales hacia los hijos. En la práctica estos factores son de especial importancia pues se trata de elementos considerados como "dinámicos" y que pueden ser modificados a través de intervenciones psicológicas tanto a nivel individual, como familiar. Sin embargo, considerar el análisis de otros factores personales y familiares asociados, como la autoestima, los estilos de enfrentamiento, la depresión, la incidencia de violencia familiar, entre otros, es recomendable en futuros estudios para poder comprender esta problemática de manera más integral y contribuir a la prevención de la ideación suicida en los jóvenes. 


\section{REFERENCIAS}

I Aguilar, J., Sarmiento, C., Valencia, A. \& Romero, L. (2007). La autoestima y sus relaciones con los estilos de crianza, las percepciones parentales y la motivación escolar. En J. Aguilar, A. Valencia y C. Sarmiento (Eds.): Relaciones familiares y ajuste personal, escolar y social en la adolescencia. Investigaciones entre estudiantes de escuelas públicas (pp. 125-138). México: Universidad Nacional Autónoma de México.

I Aguirre-Flórez, D. C., Cataño-Castrillón, J. J., Cañón, S.C., Marín-Sánchez, D. F., Rodríguez-Pabón, J. T., Rosero-Pantoja, L. A., Valenzuela-Díaz, L. P., \& Vélez-Restrepo, J. (2014). Riesgo suicida y factores asociados en adolescentes de tres colegios de la ciudad de Manizales (Colombia). Revista de la Facultad de Medicina, 63(3), 419-429. http://dx.doi.org/10.15446/revfacmed.v63n3.44205

I Andrade, J. A. (2012). Aspectos psicosociales del comportamiento suicida en adolescentes. Revista Electrónica de Psicología Iztacala, 12(2), 688-721.

I Andrade, J. A., Bonilla, L. \& Valencia, Z. (2010). Pretective Factors of Suicidal Ideation in 50 Women from Prison: Villa Cristina - Armenia-Quindío (Colombia). Revista Orbis, 6(17), 6-32.

I Andrade, S. J. A. \& Gonzáles, P. J. (2017). Relación entre riesgo suicida, autoestima, desesperanza y estilos de socialización parental en estudiantes de bachillerato. Psicogente, 20(37), 70-88. https://doi. org/10.17081/psico.20.37.2419

I Bahamon Muñetón, J., \& Alarcón-Vázquez, Y. (2018). Diseño y validación de una escala para evaluar el Riesgo Suicida (ERS) en adolescentes colombianos. Universitas Psychologica, 17(4), 1-15. https://doi. org/10.11144/Javeriana.upsy17-4.dvee

I Balam, L. (2019, febrero 18). Repuntan casos de suicidio entre menores en Yucatán. "La Jornada Maya". Recuperado de https://www.lajornadamaya.mx/2019-02-18/Repuntan-casos-de-suicidio-entre-menoresen-Yucatan

I Borges, G., Orozco, R., Villatoro, J., Medina-Mora, M. E., Fleiz, C., \& Díaz-Salazar, J. (2019). Suicide ideation and behavior in Mexico: ENCODAT 2016. Salud Pública de México, 61, 6-15.

1 Cabra, O., Infante, D. \& Sosa, F. (2010). El suicidio y los factores de riesgo asociados en niños y adolescentes. Revista Médica Sanitas, 13(2), 28-35.

I Capano, B. A., González, T., M. del L. \& Massonnier, N. (2016). Estilos relacionales parentales: estudio con adolescentes y padres. Revista de Psicología (PUCP), 34(2), 413-444. http://dx.doi.org/10.18800/ psico. 201602.008

I Casais, M. D. \& Flores, G. M. (2017). Prácticas parentales como determinantes de la motivación al logro y evitación al éxito en adolescentes. En M. Flores G., M. L. Cortés A. y M. T. Morales M. (Comp.) Estudios sobre la crianza en México (pp.151-186). Mérida, Yucatán: Universidad Autónoma de Yucatán.

I Chávez-Hernández, A. M., González-Forteza, C., Juárez, L. A., Vázquez, V. D. Y Jiménez, T. A. (2015). Ideación y tentativas suicidas en estudiantes de nivel medio del estado de Guanajuato, México. Acta Universitaria, 25, 43-50.

1 https://doi.org/10.15174/au.2015.786

$\checkmark$ Código Ético del Psicólogo (2002). México: Sociedad Mexicana de Psicología. 
I Cohen, J. (1989). Statistical Power Analysis for the Behavioral Sciences (2 $2^{\mathrm{a}}$ ed.). New York: EEUU Erlbaum.

I Córdoba, M. A., Cubillas, M. J. \& Román, R. (2011). ¿Es posible prevenir el suicidio? Evaluación de un programa de prevención en estudiantes de bachillerato. Pensamiento Psicológico, 9(17), 21-32.

$\checkmark$ Córdova, A., Estrada, A. \& Velázquez, M. (2013). Detección de riesgo suicida en una muestra de adolescentes estudiantes de escuelas secundarias. Revista Intercontinental de Psicología y Educación, $15(1), 7-21$.

\ Cortés, A. L., Flores, G. M. \& Morales, M. M. T. (2017). Percepción de la crianza paterna: su relación con la empatía e inteligencia emocional. En M. Flores G., M. L. Cortés A. y M. T. Morales M. (Comp.) Estudios sobre la crianza en México (pp. 57-98). Mérida, Yucatán: Universidad Autónoma de Yucatán.

I Etxebarria, I., Apodaca, P., Fuentes, M., López, F. \& Ortíz, M. J. (2009). La crianza y la educación de los hijos en la sociedad actual ¿lo estamos haciendo bien? Revista de Psicología Social, 24(1), 1-16. https:// doi.org/10.1174/021347409786922952

I Florenzano, R., Valdez, C. M., Cáceres, C. E., Santander, R. S., Aspillaga, H. C. \& Musalema, C. (2011). Relación entre ideación suicida y estilos parentales en un grupo de adolescentes chilenos. Revista Médica de Chile, 139, 1529-1533. http://doi.org/10.4067/S0034-98872011001200001

I Flores, G. M. \& Cortés, A. L. (2017). Validación de una escala de percepción de prácticas de crianza. En, M. Flores G., M. L. Cortés A. y M. T. Morales M. (Comp.) Estudios sobre la crianza en México (pp. 22-56) Mérida, Yucatán: Universidad Autónoma de Yucatán.

I Flores, G. M., Cortés, A. L., Morales, M. M. T., Campos, M. M. J. \& Díaz-Loving, R. (2019). Crianza materna y regulación emocional en adolescentes: diferencias por sexo. Emerging Trend in Education, 1(2), 96-120. https://doi.org/10.19136/etie.a1n2.2781

I Forero, I., Sibato, E. \& Salamanca, Y (2018) Ideación suicida, funcionalidad familiar y consumo de alcohol en adolescentes de Colombia. Revista Latinoamericana de Ciencias Sociales, Niñez y Juventud, 15(1), 431-442. http://dx.doi.org/10.11600/1692715x.1512729042016.

I Fuentes, M. C., García, E., Gracia, F. \& Alarcón, A. (2015). Los estilos parentales de socialización y el ajuste psicológico. Un estudio con adolescentes españoles. Revista de Psicodidáctica, 20(1), 117-138. http://dx.doi.org/10.1387/RevPsicodidact.10876

$\checkmark$ Garlow, S. J., Rosenberg, J., Moore, J.D., Hass, A. P., Koestner, B., Hendin, H., \& Nemeroff, C. B. (2008). Depression, desperation, and suicidal ideation in college students: Results from de American Foundation for Suicide Prevention College Screenin Project at Emory University. Depression and Anxiety, 25, 428488. https://doi.org/10.1002/da.20321

I Gibb, S. J., Beautrais, A. L. \& Fergisson, D. M. (2005). Mortality and further suicidal behavior after an index suicide attempt: a 10 years study. Australian \& New Zeland Journal of Psychiatry, 39(1-2), 95-100. https://doi.org/10.1080/j.1440-1614.2005.01514.x

I González, C., Ramos, L. Caballero, M. \& Wagner, F. (2003). Correlatos psicosociales de depresión, ideación e intento suicida en adolescentes mexicanos. Psicothema, 15(4), 524-532. 
I Hernández, M. L., de los Reyes, A. G. González, Y.A. \& Prats, J. C. (2015). Caracterizacio de los adolescentes comisores del intento sicida del Consejo Popular Siboney. Ocutbre de 2010 a marzo de 2011. Multimed,19, 1-10.

I Jacob Foundation, Hanbury, C. \& Malti, T. (2011). Monitoring and evaluation life skills for youth development. Vol 1: The Guidelines. Zurich: Layout Press/Printing.

I Kim, B. Y., \& Lee, C. S. (2009). A Meta-Analysis of Variables Related to Suicidal Ideation in Adolescents. Journal of Korean Academy of Nursing, 39(5), 651-661. https://doi.org/10.4040/ jkan.2009.39.5.651

I Lai, K.W. \& Mcbride-Chang, C. (2001). Suicidial Ideation, Parenting Style and Family Climate among Hong Kong Adolescents. International Journal of Psychology, 36, 2, 81-87. https://doi. org $/ 10.1080 / 00207590042000065$

$\checkmark$ Loboa, R. N. J. \& Morales, O. D. F. (2016). Perfil de orientación al suicidio en adolescentes escolarizados, Villahermosa-Tolima, 2013. Revista Facultad Nacional de Salud Pública, 34(1), 94-102. https://doi.org/ 10.17533/udea.rfnsp.v34n1a12

I Lord, F. M. \& Novick, M. R. (1968). Statistical theories of mental test scores. Menlo Park: AddisonWesley.

$\checkmark$ McAuliffe, C.M. (2002). Suicidal Ideation as an Articulation of Intent: A focus for Suicide Prevention? Archives of Suicide Research, 6(4), 325-338. https://doi.org/10.1080/13811110290114106

$\checkmark$ Morales, S., Armijo, I., Moya, C., Echávarri, O., Barros, J. Varela, C., Fischman, R., Peñaloza, F. \& Sánchez, G. (2014). Avances en Psicología Latinoamericana, 32(3), 403-417.

I Morales, S., Echávarri, O., Barros, J. \& Maino, M. de la P. (2017). Intento e ideación suicida en consultantes de salud mental: estilos depresivos, malestar interpersonal y satisfacción familiar. PSYKHE, 26(1), 1-14. http://dx.doi.org/10.7764/psykhe.26.1.939

I Mustanski, B. \& Liu, R. T. (2013). A longitudinal study of predictors of suicide attempt among lesbian, gay, bi-sexual and transgender youth. Archives of Sexual Behavior, 42(3), 437-448. https://doi.org/10.1007/ s10508-012-0013-9

I Nock, M. K., Borges, G., Bromet, E. J., Cha, C.B., Kessler, R.C. \& Lee, S. (2008). Suicide and suicidal behavior. Epidemiologic Reviews, 30(1), 133-54. https://doi.org/10.1093/epirev/mxn002

I Organización Mundial de la Salud. (2012). El suicidio, un problema de salud pública enorme y, sin embargo, prevenible. Ginebra: OMS

I Organización Mundial de la Salud. (2014). Prevención del suicidio, un imperativo global. Ginebra: OMS

I Parker, H. \& McNally, R. (2008). Repressive coping, emotional adjustment an cognition in people who have lost loves ones to suicide. Suicide Life Theatening Behavior, 38(6), 676-687.

I Parra, A. \& Oliva, A. (2002). Comunicación y conflicto familiar durante la adolescencia. Anales de Psicología, 18(2), 215-231.

I Pérez, A. P., Uribe, I., Vianchá, M., Bahamón, M. Verdugo, J. C. \& Ochoa, S. (2013). Estilos parentales como predictores de ideación suicida. Psicología desde el Caribe, 30(3), 551-568. 
I Pérez, I., Ibañez, M. \& Reyes, J. (2008). Factores asociados al intento suicida e ideación suicida persistente en un Centro de Atención Primaria. Bogota 2004-2006. Revista Salud Publica, 10(3), 374-385.

I Pinto, L., Silva-León, A. M. \& Moore, V. I. (2009). Características psicosociales de los adolescentes yucatecos con y sin conducta suicida. Educación y Ciencia, 1(1-36), 71-84.

I Pitman, A. L., Osborn, D. P. J., Rantell, K., \& King, M. B. (2016). Bereavement by suicide as a risk factor for suicide attempt: a cross-sectional national UK-wide study of 3432 young bereaved adults. BMJ Open, 6, 1-12. http://doi.org/10.1136/bmjopen-2015-009948

I Plutchik R., Van Praag H., Conte H.R. \& Picard, S. (1989). Correlates of suicide and violence risk, I: The suicide risk measure. Comprehensive Psychiatry, 30, 296-302. https://doi.org/10.1016/0010440X(89)90053-9

I Quintanilla-Montoya, R., Sánchez,-Loyo, L. M., Cruz-Gaytán, J. L., Benítez-Pérez, L. E., Morfín-López, T., García de Alba-García, J. E., Aranda-Mendoza, A. J. \& Vega-Miranda, J. C. (2015). Del contexto cultural al funcionamiento familiar. Conocimientos compartidos y relaciones familiarea en adolescnetes con intento de suicidio. Educacion y Desarrollo, 33, 15-26.

I Rhodes, A. E., Boyle, M. H., Bethell, J., Wekerle, C. Goodman, D., Tonmyr, L. \& Manion, I. (2012). Child maltratment and onset of emergency department presentations for suicide-related behaviors. Child Abuse and Neglect, 36(6), 542-551. https://doi.org/10.1016/j.chiabu.2012.04.006

I Robledo, A. (2007). Suicide in adolescents: what the equipment of health must know. Revista Peruana de Pediatría, 60(1), 61-64.

I Rosales J.C., Córdova M., \& Fonseca M.P. (2013). Ideación suicida en estudiantes de la Universidad Tecnológica del Estado de Tlaxcala y variables asociadas. Alternativas en Psicología, 28, 20-32.

I Rosales, P. J. C., Córdoba, O. M. \& Guerrero, B. D. I. (2013). Ideación suicida y variables asociadas conforme al sexo, en estudiantes de la Universidad Tecnológica del estado de Puebla, México. Revista Electrónica de Psicologia Iztacala, 16, 233-243.

I Rubio, G, Montero, I., Jáuregui, J., Villanueva, R., Casado, M.A., Marín J.J. \& Santo-Domingo, J. (1998). Validación de la escala de riesgo suicida de Plutchik en población española. Archivos de Neurobiología, 61, 143-52.

I Sánchez Sosa, J.J., Villareal-González, M. E., Musitu, G. \& Martínez, F. B. (2010). Ideación suicida en adolescentes: Un análisis psicosocial. Intervención Psicosocial, 9(3), 279-287. https://doi.org/10.5093/ in2010v19n3a8

ISánchez, R., Cáceres, H. \& Gómez, D. (2002). Ideación suicida en adolescentes universitarios: Prevalencia y factores asociados. Biomédica 22(2), 407-416

I Sánchez-Teruel, D. \& Robles Bello, M. A. (2014). Factores protectores que promueven la resiliencia ante el suicidio en adolescentes y jóvenes. Papeles del Psicólogo, 35(3), 181-192.

I Sánchez-Teruel, D. (2012). Variables sociodemográficas y biopsicosociales relacionadas con la conducta suicida. En J. A. Muela, A. García y A. Medina (eds.). Perspectivas en Psicología Aplicada (pp. 61-78). Jaén: Centro Asociado Andrés de Vandelvira de la UNED. 
I Sánchez-Teruel, D., García-León, A. \& Muela-Martínez, J. A. (2013). Relación entre alta ideación suicida y variables psicosociales en estudiantes universitarios. Electronic Journal of Research in Educational Psychology, 11(2), 429-450.

I Santana-Campas, M. A., \& Santoyo Telles, F. (2018). Propiedades psicométricas de la Escala de Riesgo Suicida de Plutchik en una muestra de jóvenes mexicanos privados de la libertad. Avances en Psicología, 26(1), 57-64. https://doi.org/10.33539/avpsicol.2018.v26n2.1127

I Sarmiento, S. C. \& Aguilar Villalobos, J. (2011). Predictores familiares y personales de la ideación suicida en adolescentes. Psicologia y Salud, 21(1), 25-30.

I Say, G. (2020). Prefectionism in Major Depressive Disorder. Psikiyatride Güncel Yaklaşımlar-Current Approaches in Psychiatry, 12(4), 562-574. https://doi.org/10.18863/pgy.703132

I Schilling, E.A., Aseltine, Jr. R.H., Glanovsky, J. L., James, A. \& Jacobs, D. (2009). Adolescent alcohol use, suicidal ideation, and suicide attempts. Journal of Adolescent Health, 44(4), 335-41. https://doi. org/10.1016/j.jadohealth.2008.08.006

I Smith, M. M., Sherry, S. B., Chen, S., Saklofske, D. H., Mushquash, C., Flett, G. L., \& Hewitt, P. L. (2018). The perniciousness of perfectionism: A meta-analytic review of the perfectionism-suicide relationship. Journal of Personality, 86(3), 522-542. https://doi.org/10.1111/jopy.12333

I Stengel E. (1987). Psicología del suicidio y los intentos suicidas. Buenos Aires: Paidós.

I The jamovi project (2020). jamovi (Versión 1.2) [Software]. Recuperado de https://www.jamovi.org

I Toro, D., Paniagua, R., González, C. \& Montoya, B. (2009). Caracterización de adolescentes escolarizados con riesgo de suicidios. Revista Facultad Nacional de Salud, 27(3), 302-308.

I Toro, R., Avendaño, B. \& Castrillón, D. (2016). Design and psychometric analysis of the hopelessness and suicide ideation inventory "IDIS”. International Journal of Psychological Research, 9(1), 52-63.

I Tur-Porcar, A., Mestre, M. V., Samper, P. \& Malonda, E. (2012). Crianza y agresividad de los menores: ¿es diferente la influencia del padre y de la madre? Psicothema, 24(2), 284-288.

$\checkmark$ Urzúa, M. \& Caqueo-Urízar, A. (2011). Construcción y evaluación psicométrica de una escala para pesquizar factores vinculados al comportamiento suicida en adolescentes chilenos. Universitas Psychologica, 10 (3), 721-734.

I Valdivia, M., Silva, D., Sanhueza, F., Cova, F. \& Melipillán, R. (2015). Prevalencia de intento de suicidio adolescente y factores de riesgo asociados en una comuna rural de la provincial de Concepción. Revista Médica de Chile, 43(3), 320-328. http://dx.doi.org/10.4067/S0034-98872015000300006

I Vallejo, C. A., Osorno, M. R. \& Mazadiego, I. T. (2008). Estilos parentales y sintomatología depresiva en una muestra de adolescentes veracruzanos. Enseñanza e Investigación en Psicología, 13(1), 91-105.

I Wichstrom, L. (2000). Predictors of adolescent suicide attemps: a nacionally representative longitudinal study of Norwegian adolescents. Journal of the American Academy of Child \& Adolescents Psychiatry, 39(5), 603-610. https://doi.org/10.1097/00004583-200005000-00014

I Zeifman, R. J., Antony, M. M., \& Kuo, J. R. (2020). When being imperfect just won't do: Exploring the relationship between perfectionism, emotion dysregulation, and suicidal ideation. Personality and Individual Differences, 152, 109612. https://doi.org/10.1016/j.paid.2019.109612 
Envío a dictamen: 8 de junio de 2020

Reenvío: 27 de junio de 2020

Aprobación: 7 de Julio de 2020

Lourdes Cortés-Ayala. Doctora en Psicología. Profesor Titular C de Tiempo Completo de la Facultad de Psicología de la Universidad Autónoma de Yucatán. Docente e Investigadora. Pertenece al SNI, Nivel 1. Correo electrónico: lourdes.cortes@correo.uady.mx

Julio Isaac Vega Cauich. Maestro en Investigación Educativay Especialista en Estadística. Es Profesor Asociado de la Facultad de Educación de la Universidad Autónoma de Yucatán, y Coordinador de Investigación de Foco Rojo: Centro de Ciencias Sociales para la Construcción de la Paz A.C. Correo electrónico: julio.vega@correo.uady.mx

Sergio Daniel Garma. Licenciado en Psicología. Maestría en Psicoterapia Psicoanalítica Relacional. Correo electrónico: sergio.psic.95@gmail.com

José Albero López Vadillo. Licenciado en Psicología. Actualmente se desempeña en Reclutamiento, Selección y Desarrollo Humano en empresa privada. Correo electrónico: albertolopez90@hotmail.com 